\title{
In utero Exposure to Cigarette Smoking, Environmental Tobacco Smoke and Reproductive Hormones in US Girls Approaching Puberty
}

\author{
Audra L. Gollenberg ${ }^{a} \quad$ O. Yaw Addo ${ }^{b}$ Zhiwei Zhang ${ }^{c}$ Mary L. Hediger ${ }^{d}$ \\ John H. Himes ${ }^{e}$ Peter A. Lee ${ }^{f, g}$ \\ a Public Health Program, College of Arts and Sciences, Shenandoah University, Winchester, Va., ${ }^{b}$ Hubert Department \\ of Global Health, Rollins School of Public Health, Emory University, Atlanta, Ga., 'Division of Biostatistics, Office \\ of Surveillance and Biometrics, Center for Devices and Radiological Health, Food and Drug Administration, Silver

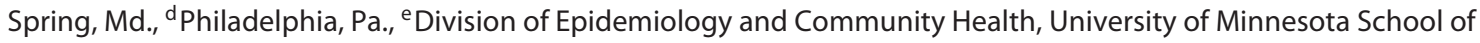 \\ Public Health, Minneapolis, Minn., ${ }^{\mathrm{f}}$ Department of Pediatrics, Penn State College of Medicine, The Milton S. Hershey \\ Medical Center, Hershey, Pa., and 9 Department of Pediatrics, Indiana University School of Medicine, Indianapolis, \\ Ind., USA
}

\section{Established Facts}

- Environmental factors have been shown to influence age at onset of puberty in girls, and this phenomenon may explain, in part, secular changes in population-based pubertal attainment.

- Maternal smoking has been explored in relation to pubertal development in girls, but results are conflicting with regard to the directionality of results.

\section{Novel Insights}

- We observed that maternal smoking was negatively associated with reproductive hormones, luteinizing hormone and inhibin B in 6- to 11-year-old girls after adjusting for multiple pathways by which early life factors may influence hormones and current exposure to environmental tobacco smoke.

- Early life tobacco smoke exposure may influence reproductive development as manifested by altered hormone levels in adolescence and should be considered in the context of life course epidemiology.

\section{Key Words}

Endocrine disruptors · Puberty · Tobacco · Adolescence ·

Environmental tobacco smoke

\begin{abstract}
Background/Aims: Evidence is unclear whether prenatal smoking affects age at menarche and pubertal development, and its impact upon hormones has not been well studied. We aim to identify potential pathways through which prenatal
\end{abstract}

\section{KARGER 125}

E-Mail karger@karger.com www.karger.com/hrp
Dr. Audra L. Gollenberg, PhD

Public Health Program

College of Arts \& Sciences, Shenandoah University

Winchester, VA 22601 (USA)

E-Mail agollenb@su.edu 
smoking and environmental tobacco smoke (ETS) affect reproductive hormones in girls approaching puberty. Methods: We examined the association between prenatal smoking, current ETS and luteinizing hormone (LH) and inhibin B $(\operatorname{InB})$ in 6- to 11-year-old girls in the 3rd National Health and Nutrition Examination Survey, 1988-1994. Parents/guardians completed interviewer-assisted questionnaires on health and demographics at the time of physical examination. Residual blood samples were analyzed for reproductive hormones in 2008. Results: Of 660 girls, 19 and 39\% were exposed to prenatal smoke and current ETS, respectively. Accounting for multiple pathways in structural equation models, prenatally exposed girls had significantly lower $\mathrm{LH}(\beta=$ $-0.205 \mathrm{log}-\mathrm{mlU} / \mathrm{ml}, \mathrm{p}<0.0001)$ and $\ln B(\beta=-0.162$, log-pg/ $\mathrm{ml}, \mathrm{p}<0.0001)$. Prenatal smoking also influenced $\mathrm{LH}$ positively and $\ln B$ negatively indirectly through BMI-for-age. ETS was positively associated with $\mathrm{LH}$, but not with InB. Conclusion: Exposure to maternal smoking may disrupt reproductive development manifesting in altered hormone levels near puberty.

(c) 2015 S. Karger AG, Basel

\section{Introduction}

Maternal smoking is known to be detrimental to fetal growth and development [1], and long-term effects on offspring have been documented for reduced fecundity [2] and chronic diseases [3]. Even so, rates of maternal smoking during pregnancy remain between 10 and $20 \%$ in the United States [4]. The concept of early origins of disease has received increasing attention in the last decade with regard to in utero exposures and risk of childhood and adult-onset disease [5]. However, few studies have addressed early life factors associated with pubertal development and reproductive hormones, even though age at onset of pubertal development is recognized as a proxy of the reproductive health of a population [6].

Environmental factors have been found to effect changes in the onset of puberty [7], highlighting the importance of environmental endocrine disruptors in influencing reproductive development. Identifying factors that influence pubertal development is important at the population level because age at puberty is associated with age at debut of sexual activity, alcohol and drug use, depression, fragility fractures and risk of chronic disease [8-10]. Further, age at menarche has been associated most notably with breast cancer [11], but may simply be an early marker of chronic estrogen exposure, endocrine disruption and/or reproductive dysfunction.

Maternal Smoking, ETS and Hormones Near Puberty
Only a few studies have examined maternal smoking in relation to pubertal development, and the results are conflicting [12-19]. Moreover, most studies were restricted to age at menarche, which occurs relatively late in the course of pubertal development and may be an insensitive indicator of pubertal onset. Individual assays of reproductive hormones may add new insight into the specific endocrine disruption associated with prenatal exposure to tobacco smoke, and the mechanisms by which early-life exposures affect reproductive development. Recent work suggests that inhibin B ( InB) and luteinizing hormone (LH) concentrations are consistent with onset of puberty as indicated by Tanner breast stage 2 and rise progressively through the pubertal stages in this population of young girls (age $8-11$ years) [20]. InB is produced by granulosa cells in the ovary paralleling development of ovarian follicles and is found to increase as girls approach puberty from 5 to 12 years of age $[21,22]$, whilst LH has long been recognized as important in the regulation of the hypothalamic-pituitary-gonadal axis and as a marker of pubertal development [22, 23]. However, previous population-based studies have not focused on hormonal markers of pubertal development.

We aimed to extend existing literature on age at menarche by measuring hormonal markers of early pubertal development that may be affected by environmental endocrine disruptors in a nationally representative population of US girls. We sought to answer the following research question: which potential pathways are important in linking prenatal smoking and current environmental tobacco smoke (ETS) with reproductive hormones in young girls? To answer this question, InB and $\mathrm{LH}$ were chosen because of their patterns in younger children and likelihood of detection in those approaching puberty.

\section{Materials and Methods}

We used data from girls aged 6-11 years who participated in the Third National Health and Nutrition Examination Survey (NHANES III), 1988-1994. In brief, NHANES III is a cross-sectional nationally representative study with a clustered, probability sampling design. Non-Hispanic Blacks and Mexican-Americans were oversampled to provide more precise estimates for these groups, and children were sampled into the following age categories: $<6,6-11$, and $12-19$ years. Details of the study design and data collection methods have been published elsewhere [24]. Youth participants $(<12$ years) consented by proxy before participating in NHANES III. IRB approval was obtained through the National Center for Health Statistics.

We included 705 (44\%) of the 1,589 girls aged $6-11$ years who participated in the household interview, had a physical exami-

Horm Res Paediatr 2015;83:36-44 
nation [24], and had residual stored blood for laboratory analysis. The main exposures were tobacco smoke in utero and current ETS. During the NHANES III interview, mothers reported if they had smoked while pregnant with the child enrolled and if they smoked throughout the entire pregnancy or during what pregnancy month they quit. The protocol did not include asking the number of cigarettes smoked per day. For assessment of ETS, mothers reported the number of cigarette smokers who currently live in the child's home. We excluded 2 girls who reported being active smokers from the main analyses.

Sociodemographic variables of interest were available from the NHANES III database and included race/ethnicity, census region, metropolitan/nonmetropolitan residence, and the poverty-income ratio (PIR). Based on the PIR distribution (range 0-6.9; median $=1.3$ ), we categorized PIR in approximate tertiles as $<1,1-2$, and $>2$, so that a higher PIR indicates better household financial status. Anthropometric measurements [height, waist circumference, body mass index (BMI, $\mathrm{kg} / \mathrm{m}^{2}$; $\mathrm{z}$-score for age and raw value)] were also considered as potential confounders. We assessed whether adjustment for timing of the blood draw (morning, afternoon, evening) altered the association between smoke exposure and found this was not so. Additional confounding and potential mediator variables that were considered were maternal age at child's birth, weekly exercise, self-reported general health status of the child (5-point scale), and birth weight as reported by the mother.

\section{Laboratory Analysis}

The laboratory assays for this project have been previously described in detail [25]. In brief, hormonal assays ( $\mathrm{InB}$ and $\mathrm{LH}$ ) were performed on nonfasting serum samples that were stored at $-70^{\circ} \mathrm{C}$ and were obtained from the National Center for Health Statistics/ Centers for Disease Control and Prevention by Rules-Based Medicine Inc. Blood was drawn at the time of clinical examination according to the NHANES III protocol and categorized as to the timing of blood draw (morning, 8 a.m. to 12 p.m.; afternoon, 12 p.m. to 4 p.m., and evening, 4 p.m. to 7 p.m.).

Specifically, InB was measured using the DSL-10-84100 ACTIVE ${ }^{\circledR}$ Inhibin B ELISA assay (Gen I assay) [26]. The functional sensitivity was a priori set at $7 \mathrm{pg} / \mathrm{ml}$ [i.e. limit of detection (LOD)] per the manufacturer instructions. LH was measured using the LH ELISA kit (Bio-Quant BQ049F), a solid phase direct sandwich method. The LOD was set at $0.05 \mathrm{mIU} /$ $\mathrm{ml}$, and values below these levels were uniformly substituted with $0.035 \mathrm{mIU} / \mathrm{ml}$, equivalent to $\mathrm{LOD} / \sqrt{ } 2$. We used the continuous form of each hormone variable to avoid any assumptions concerning puberty-related hormone thresholds and the known interindividual and intraindividual variation in hormone concentrations.

\section{Statistical Methods}

We categorized in utero exposure to smoking dichotomously (yes/no) and categorized current ETS exposure by number of smokers in the home $(0,1$, and $2+)$. Both descriptive and analytic techniques were used to inspect and assess the data for the 705 girls with complete LH hormone measurements, and of these, 689 (98\%) had complete InB analysis. We created newly derived inverse probability sampling weights [27] to account for NHANES III's complex sampling design and the limited sera available for hormone analyses. Data management was conducted with SAS v9.3 (SAS, Cary, N.C., USA) and Structural Equation Models were built using Analysis of Moment Structures software (AMOS, IBMSPSS, Chicago, Ill., USA).

\section{Structural Equation Modeling}

We used recursive structural equation modeling (SEM) to examine hypothesized causal relations between observed and unobserved/latent variables with comprehensive modeling measurement errors [28]. This approach is well-suited for our research question - an environmental risk factor and later biological outcome [29] and the left censored hormone data available. We also explored the SEMs' ability to model latent effects to address the LOD problem with hormone measures. As a result, the LOD was modeled as a latent factor in all SEMs while accommodating data challenges associated with environmental epidemiological data [30]. Bayesian SEM techniques were utilized to obtain robust estimation of pathway parameters while handling both categorical and continuous variables efficiently $[31,32]$.

We a priori identified potential SEM directional pathways predictive of puberty and hormone levels based on prior literature [14] and aimed to keep models as parsimonious as possible. Primary pathways are presented in figure 1 , including direct pathways between each smoking exposure to the hormones or indirectly affecting birth weight and later body size. For conceptual reasons, we also examined the joint effects of maternal smoking and ETS using an interaction term of maternal smoking $\times$ ETS as a separate pathway, as mothers who smoke during pregnancy may be more likely to continue smoking postnatally and/or live with other smokers.

Statistical significance of free parameters was formally tested using regression estimates to be different from zero with two-sided $p$ values $(<0.05)$. We also accounted for both observed and unobserved confounders through alternate structural pathways operating distally to influence hormone levels. They included PIR, sampling region, race/ethnicity, maternal age, child's age at blood draw, and general health status.

Several sensitivity analyses were conducted. To assess the effect of ETS alone on hormones, we constructed an SEM among only girls who were unexposed to tobacco in utero $(n=556)$. We also ran the full analysis after excluding the small group of menarcheal girls $(n=33)$ due to potential hormonal variations.

\section{Results}

Approximately $19 \%(\mathrm{n}=126)$ of girls were exposed to maternal smoking in utero. Of these, 95 (75\%) girls were exposed to maternal smoking throughout the pregnancy, whereas 31 girls (25\%) were exposed during part of the pregnancy. A rather high proportion of girls were currently exposed to ETS in the home by maternal report of $1+$ smokers living with the child (40\%), and of these, 100 (15\%) girls were exposed to both in utero tobacco smoke and ETS.

Table 1 displays characteristics of participants by maternal smoking status. Most maternal and child characteristics were not significantly associated with exposure 
Fig. 1. Structural equation model pathways linking maternal smoking, environmental tobacco smoke, and hormones in girls. All path variables (observed and latent) have been simultaneously adjusted for measurement error terms. BMI = Body mass index; ETS = environmental tobacco smoke; LOD = limit of detection; PIR = povertyincome ratio.

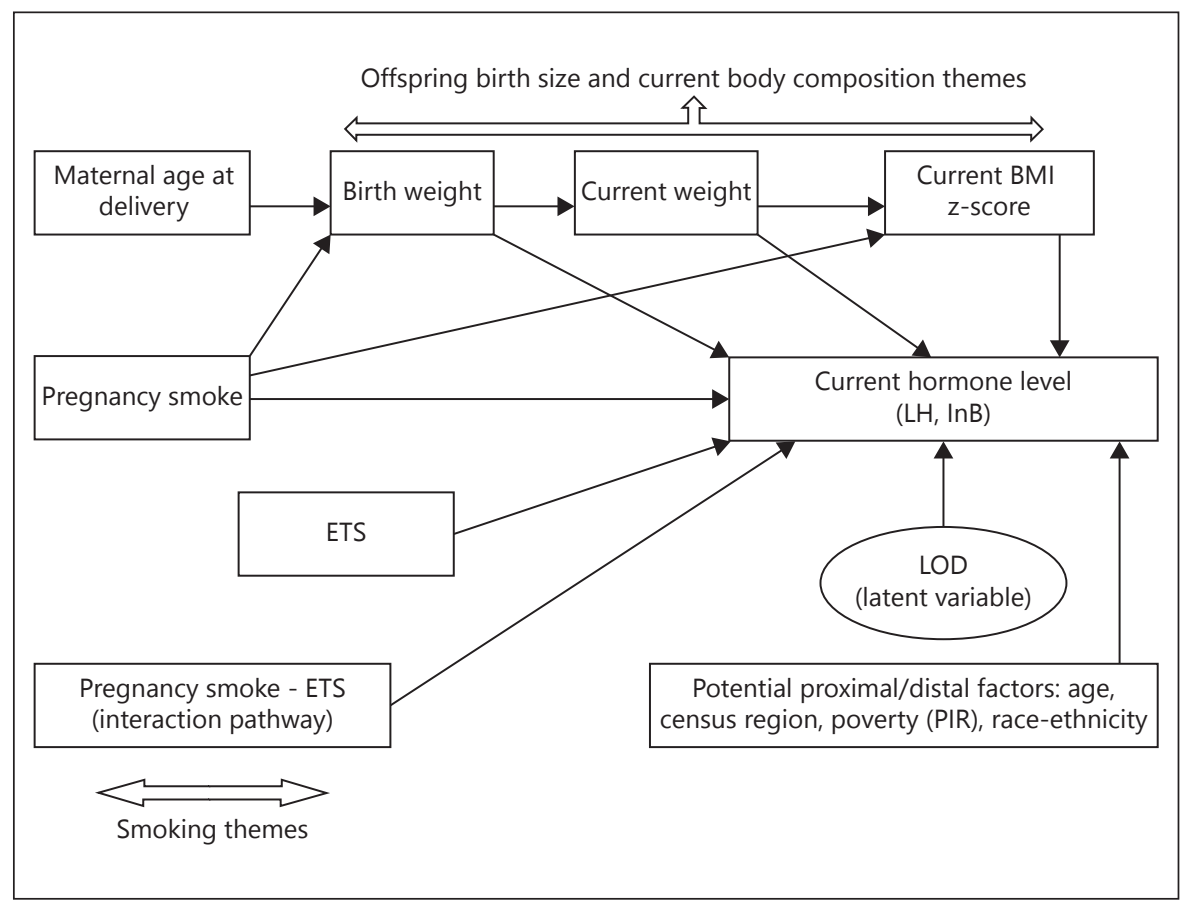

to maternal smoking. A few factors were significant $(\mathrm{p}<$ 0.01): race/ethnicity, number of smokers in the house, maternal age, geographic region and weekly exercise. In terms of anthropometry, girls exposed to maternal smoke had greater waist circumferences $(p=0.006)$ and were more often low birth weight (10.6 vs. $7.7 \%$, nonsignificant) than those unexposed. Further, this subset of 6- to 11-year-old girls from NHANES III with stored sera was compared to the full NHANES III sample with regard to sociodemographics and hematologic and anthropometric measurements [25]. As described previously, few statistically significant differences were noted between the NHANES III sample with stored sera and those without. The noted exceptions were fewer non-Hispanic white girls and a slightly younger age by 4 months in this analytic sample. These differences were expected to occur by chance due to limited availability of sera.

The SEM estimates are displayed in table 2 as they relate to LH specifically. We found that maternal smoking exposure was associated with decreased LH ( $p<0.0001)$, after accounting for confounders, mediators, and unobserved error, whereas current ETS exposure was positively associated with $\mathrm{LH}(\mathrm{p}<0.0001)$ with a negative interaction term (maternal smoking $\times$ ETS: $p<0.0001$ ). Further, maternal smoking was also significantly associated with LH indirectly through the BMI-for-age pathway; maternal smoking was associated with increased BMI-for-age

Maternal Smoking, ETS and Hormones Near Puberty and this in turn was associated with increased LH (table 2). Maternal smoking was negatively associated with birth weight by approximately $150 \mathrm{~g}$ ( $\mathrm{p}<0.0001$; data not shown) and the child's age (months) at blood draw was positively associated with LH $(\mathrm{p}<0.0001)$. Finally, when the model was restricted to girls who were not exposed to tobacco smoke in utero, ETS still remained significantly positively associated with LH (data not shown).

When examining the relationship between maternal smoking, ETS exposure and InB, a similar result was observed (table 3 ). In the presence of a statistically significant interaction term of maternal smoking $\times$ ETS $(\mathrm{p}<$ $0.0001)$, maternal smoking was associated with decreased InB $(\mathrm{p}<0.0001)$, whereas ETS was not associated with InB (table 3 ). In addition, a statistically significant indirect pathway was observed through which maternal smoking was significantly associated with InB indirectly through BMI-for-age. More specifically, maternal smoking was associated with increased BMI-for-age ( $\mathrm{p}<$ 0.0001 ) and BMI-for-age was significantly associated with decreased InB $(\mathrm{p}<0.0001)$. Finally, in the sensitivity analysis among only girls not exposed to tobacco smoke in utero, ETS was not significantly associated with InB.

When the SEMs were run among only premenarcheal girls, results remained robust and did not significantly change in direction, magnitude, or statistical significance. 
Table 1. Participant characteristics according to maternal smoking exposure, NHANES III, 1988-1994

\begin{tabular}{|c|c|c|c|}
\hline \multirow[t]{2}{*}{ Characteristics } & \multicolumn{2}{|c|}{ Exposure to maternal smoking } & \multirow[t]{2}{*}{$\mathrm{p}$ value } \\
\hline & No & Yes & \\
\hline Participants & $534(80.8)$ & $127(19.2)$ & \\
\hline \multicolumn{4}{|l|}{ Race/ethnicity } \\
\hline White & $100(18.7)$ & $45(35.4)$ & \multirow[t]{3}{*}{$<0.001$} \\
\hline Black & $194(36.3)$ & $58(45.7)$ & \\
\hline Mexican & $240(44.9)$ & $24(18.9)$ & \\
\hline \multicolumn{4}{|l|}{ PIR } \\
\hline$<1$ & $212(39.7)$ & $51(40.2)$ & \multirow[t]{4}{*}{0.98} \\
\hline $1-2$ & $127(23.8)$ & $32(25.2)$ & \\
\hline$>2$ & $156(29.2)$ & $35(27.6)$ & \\
\hline Don’t know & $39(7.3)$ & $9(7.1)$ & \\
\hline \multicolumn{4}{|l|}{ Sampling region } \\
\hline Northeast & $41(7.7)$ & $7(5.5)$ & \multirow[t]{4}{*}{0.008} \\
\hline Midwest & $110(20.6)$ & $40(31.5)$ & \\
\hline South & $206(38.6)$ & $54(42.5)$ & \\
\hline West & $177(33.1)$ & $26(20.5)$ & \\
\hline \multicolumn{4}{|l|}{ Residence } \\
\hline Metropolitan & $289(54.1)$ & $62(48.8)$ & \multirow[t]{2}{*}{0.28} \\
\hline Nonmetropolitan & $245(45.9)$ & $65(51.2)$ & \\
\hline \multicolumn{4}{|l|}{ Low birth weight } \\
\hline No & $471(92.4)$ & $110(89.4)$ & \multirow[t]{2}{*}{0.29} \\
\hline Yes & $39(7.7)$ & $13(10.6)$ & \\
\hline \multicolumn{4}{|c|}{ Sweat during exercise: times per week } \\
\hline $0-1$ & $198(37.1)$ & $46(36.2)$ & \multirow[t]{4}{*}{0.04} \\
\hline $1-2$ & $94(17.6)$ & $11(8.6)$ & \\
\hline $3-5$ & $147(27.5)$ & $42(33.1)$ & \\
\hline 6 or more & $95(17.8)$ & $28(22.1)$ & \\
\hline \multicolumn{4}{|l|}{ General health rating } \\
\hline Good/excellent & $476(89.1)$ & $118(92.9)$ & \multirow[t]{2}{*}{0.21} \\
\hline Fair/poor & $58(10.9)$ & $9(7.1)$ & \\
\hline \multicolumn{4}{|c|}{ Number of smokers in the household } \\
\hline 0 & $374(70.0)$ & $27(21.3)$ & \multirow[t]{3}{*}{$<0.0001$} \\
\hline 1 & $121(22.7)$ & $50(39.4)$ & \\
\hline $2+$ & $39(7.3)$ & $50(39.4)$ & \\
\hline \multicolumn{4}{|l|}{ Ever smoked at least 1 cigarette } \\
\hline No & $329(97.1)$ & $78(95.1)$ & \multirow[t]{2}{*}{0.49} \\
\hline Yes $^{\mathrm{a}}$ & $10(2.9)$ & $4(4.9)$ & \\
\hline Age, months & $108.0(106.2-109.7)$ & $106.5(102.9-110.1)$ & 0.48 \\
\hline BMI z-score for age & $0.34(0.24-0.44)$ & $0.48(0.29-0.67)$ & 0.2 \\
\hline BMI value & $18.0(17.7-18.4)$ & $18.6(18.0-19.3)$ & 0.13 \\
\hline Maternal age at birth, years & $25.0(24.5-25.5)$ & $23.7(22.7-24.8)$ & 0.03 \\
\hline Height $\mathrm{z}$-score for age & $0.31(0.22-0.40)$ & $0.29(0.11-0.48)$ & 0.88 \\
\hline Waist-to-height ratio & $0.86(0.85-0.86)$ & $0.87(0.86-0.88)$ & 0.006 \\
\hline
\end{tabular}

Data are presented as $\mathrm{n}(\%)$ or mean $(95 \% \mathrm{CI})$. p values were calculated using the $\chi^{2}$ test for categorical variables and t tests for continuous variables. ${ }^{a}$ Of the 14 who reported ever smoking at least 1 cigarette, only 2 individuals reported being a current smoker.

\section{Discussion}

We observed a negative association between exposure to maternal smoking and $\mathrm{LH}$ and $\mathrm{InB}$ concentrations in 6- to 11-year-old girls, whereas current ETS exposure was associated with increased $\mathrm{LH}$, but not InB directly or in- directly. The differential timing of exposure across sensitive developmental windows between maternal smoking and childhood ETS may be a major contributing factor to the resultant effects on hormone measures, with in utero exposure potentially more harmful to reproductive development. The statistically significant interaction terms of
40 
Table 2. Model 1: primary pathways for the association between maternal smoking, ETS and LH in peripubertal girls, NHANES III, 1988-1994

\begin{tabular}{lcccc}
\hline Pathways & Estimate & SE & CR & p value \\
\hline Maternal smoking $\rightarrow$ LH & -0.205 & 0.014 & -15.03 & $<0.0001$ \\
Maternal smoking $\rightarrow$ BMI-for-age & 0.158 & 0.027 & 5.72 & $<0.0001$ \\
ETS $\rightarrow$ LH & 0.056 & 0.007 & 7.49 & $<0.0001$ \\
BMI-for-age $\rightarrow$ LH & 0.035 & 0.004 & 8.04 & $<0.0001$ \\
Maternal smoking $\times$ ETS $\rightarrow$ LH & -0.039 & 0.008 & -4.64 & $<0.0001$ \\
Offspring age $\rightarrow$ LH & 0.214 & 0.003 & 75.95 & $<0.0001$ \\
\hline
\end{tabular}

$\mathrm{SE}=$ Standard error; $\mathrm{CR}=$ critical ratio. $\mathrm{LH}$ estimates are in log-mIU/ml units. BMI-for-age estimates are in Z-score units.

Table 3. Model 2: primary pathways for the association between maternal smoking, ETS and InB in peripubertal girls, NHANES III, 1988-1994

\begin{tabular}{lcccc}
\hline Pathway & Estimate & SE & CR & p value \\
\hline Maternal smoking $\rightarrow$ InB & -0.162 & 0.022 & -7.260 & $<0.0001$ \\
Maternal smoking $\rightarrow$ BMI-for-age & 0.157 & 0.027 & 5.723 & $<0.0001$ \\
ETS $\rightarrow$ InB & 0.007 & 0.012 & 0.567 & 0.571 \\
BMI-for-age $\rightarrow$ InB & -0.098 & 0.007 & -13.642 & $<0.0001$ \\
Maternal smoking $\times$ ETS $\rightarrow$ InB & 0.082 & 0.014 & 5.972 & $<0.0001$ \\
Offspring age $\rightarrow$ InB & 0.156 & 0.005 & 33.745 & $<0.0001$ \\
\hline
\end{tabular}

InB estimates are in log-pg/ml units. BMI-for-age estimates are in $\mathrm{z}$-score units.

maternal smoking $\times$ ETS exposure may suggest an adaptive effect of dual exposures on reproductive development, and thus requires further examination in a longitudinal context. A decrease in InB, a marker of ovarian follicle development [22], and the gonadotropin LH, may suggest altered endocrine function that should be considered in the context of potential long-term chronic disease and reproductive function. This is the first study to examine this association with hormonal biomarkers for hypothalamic-pituitary-gonadal activation in young girls near the onset of puberty and to account for multiple pathways. Our study represents a moderately large, nationally representative sample of racially diverse girls in comparison to previous studies.

Few previous studies have examined maternal smoking in relation to pubertal development, and the majority was limited to an outcome of age at menarche. Factors that affect pubertal onset and endocrine function may not operate under the same mechanisms as factors that affect the rate at which girls mature through the successive pubertal stages. Further, we sought to ask a different question: what is the relationship between maternal smoking, ETS and reproductive hormone concentrations, rather than age at menarche. Results were conflicting amongst the existing menarche studies. Of the 6 studies that examined maternal smoking and age at menarche, 3 found an earlier age at menarche $[13,15,16], 2$ found a later age at menarche $[17,18]$, and 1 found no association [19] when comparing exposed versus unexposed. Two studies originating from the AVON Longitudinal Study measured pubertal development based on self-reported Tanner stages in girls age 8-14 years and found an earlier pubertal development in girls exposed to third-trimester maternal smoking compared to the unexposed $[12,14]$. Finally, the only study to measure the association between maternal smoking and reproductive hormones did so among a smaller cross-sectional sample $(n=213)$ of 14 - to 17 -year-olds [33]. These authors found no associations between maternal smoking and anti-mullerian hormone, $\mathrm{InB}$, and $\mathrm{FSH}$, though daughters exposed to maternal smoking had significantly smaller uterine volumes [33]. Furthermore, there are 
sparse data examining ETS exposure in childhood and pubertal development.

Many of the thousands of chemicals found in tobacco smoke, including metals, pesticides and polyaromatic hydrocarbons, have been found to be antiestrogenic [34] and more recently, found to affect progesterone and gonadotropins in premenopausal women, with effects differing by hormone and phase of the menstrual cycle [35]. Our study extends this knowledge by using SEMs to demonstrate that maternal smoking is structurally associated with altered hormone levels in young girls. While we are not able to determine the exact biologic mechanism by which tobacco smoke in utero may disrupt endocrine regulation, this study adds to prior literature by being among the first to demonstrate multiple pathways by which maternal smoking and its mediators may modify reproductive development. We hypothesize that in utero exposure to tobacco smoke may alter reproductive hormone levels, but the observed changes may not fully manifest until puberty approaches or even later, as other studies have observed with different, adverse early-life exposures [3638 .

There have been few studies to examine the association between childhood ETS exposure and pubertal development. The few studies that have examined ETS exposure and age at menarche did not observe a robust association $[15,18]$, though one study did observe a synergistic effect of both maternal exposure and childhood ETS exposure on a later age at menarche [18]. To date we could not identify a study that has examined both current ETS and reproductive hormones in this age group. More research is needed to elucidate the effects of ETS on reproductive development in young girls.

Our observation that maternal smoking may influence $\mathrm{InB}$ and LH levels indirectly through BMI warrants further investigation. It has been documented that in utero exposure to tobacco smoke influences early childhood growth, most often evidenced by a decrease in birth weight and a later increase in weight gain and child BMI [39]. In addition, it has been reported that girls with greater BMI often experience earlier age at menarche, a late-stage pubertal event $[18,19]$. However, it remains unclear if girls with greater childhood BMI develop breast changes as a consequence of mild increases in estrogen stimulation, actually enter puberty earlier as defined by increased hypothalamic-pituitary-ovarian activity, or instead, progress through the pubertal stages quicker, thereby experiencing the late-stage pubertal event, menarche, earlier [40]. Our results may suggest that a greater BMI at age 6-11 years of age is inversely associated with InB lev- els (table 3 ) but positively associated with LH levels (table 2), and this contrasting effect has been observed in adult fertile women and those with polycystic ovarian syndrome [41, 42]. Therefore, the relationship between maternal smoking and child growth and adiposity may differentially influence reproductive development as manifested through LH and InB pathways.

We employed a robust SEM approach which precluded our models from relying on an a priori assumption of linear association structure, but instead to examine both direct and indirect pathways through which primary exposures may be associated with pubertal hormones. SEMs connect all covariates by their moments for the estimation of associations, with explicit regard for the time sequence; unlike multiple regression techniques [30] which assume these periods/covariates occur concurrently and also cannot adequately address mediated pathways. Our use of SEMs is largely hypothesis generating; however, the SEMs are based on a sound conceptual framework and have been applied in multiple fields [29].

Our results should be interpreted in light of the limitations inherent in the cross-sectional design of NHANES III and the methods of ascertaining prenatal smoking and ETS exposure. Mothers were asked to report whether they smoked during the pregnancy with the index child and the child's birth weight, and this recall may have extended 6-11 years. Any misclassification of in utero smoke exposure or birth weight due to inaccurate maternal recall, however, would most likely be random and not related to the daughter's hormone levels. ETS exposure was also determined by parental self-report; however, previous validation studies have shown that parents in NHANES III are generally accurate in reporting child's ETS exposure as validated against cotinine measurements [43]. In addition, dose of cigarettes per day was not collected and precluded our ability to assess dose-response associations.

\section{Conclusion}

We found maternal smoking was negatively associated with $\mathrm{LH}$ and $\mathrm{InB}$, and the effect was modified by the presence of current ETS exposure. Experimental and epidemiologic evidence suggests biologic plausibility for the endocrine-disrupting properties of maternal smoking. Further research should extend the hormone analyses to later ages to relate in utero exposures and ETS to hormones, timing of secondary sexual characteristics, and age at menarche. Longitudinal studies that incorporate DOI: $10.1159 / 000369168$
Gollenberg/Addo/Zhang/Hediger/Himes/ Lee 
multiple hormone measurements tracking children as they approach and progress through pubertal stages will add further insight into the biological mechanisms linking maternal smoking and ETS to endocrine disruption in adolescents [6].

\section{Acknowledgements}

Laboratory analyses were completed with intramural support from the Eunice Kennedy Shriver National Institute of Child Health \& Human Development/National Institutes of Health.

\section{References}

1 Naeye RL: Influence of maternal cigarette smoking during pregnancy on fetal and childhood growth. Obstet Gynecol 1981;57:18-21.

- 2 Jensen TK, Henriksen TB, Hjollund NH, Scheike T, Kolstad H, Giwercman A, Ernst E, Bonde JP, Skakkebaek NE, Olsen J: Adult and prenatal exposures to tobacco smoke as risk indicators of fertility among 430 Danish couples. Am J Epidemiol 1998;148:992997.

-3 Doherty SP, Grabowski J, Hoffman C, Ng SP, Zelikoff JT: Early life insult from cigarette smoke may be predictive of chronic diseases later in life. Biomarkers 2009;14(suppl 1):97101.

- 4 Anderka M, Romitti PA, Sun L, Druschel C, Carmichael S, Shaw G; National Birth Defects Prevention Study: Patterns of tobacco exposure before and during pregnancy. Acta $\mathrm{Ob}$ stet Gynecol Scand 2010;89:505-514.

-5 Barouki R, Gluckman PD, Grandjean P, Hanson M, Heindel JJ: Developmental origins of non-communicable disease: implications for research and public health. Environ Health 2012;11:42.

-6 Buck Louis GM, Gray LE Jr, Marcus M, Ojeda SR, Pescovitz OH, Witchel SF, Sippell W, Abbott DH, Soto A, Tyl RW, Bourguignon JP, Skakkebaek NE, Swan SH, Golub MS, Wabitsch M, Toppari J, Euling SY: Environmental factors and puberty timing: expert panel research needs. Pediatrics 2008; 121(suppl 3):S192-S207.

-7 Euling SY, Selevan SG, Pescovitz OH, Skakkebaek NE: Role of environmental factors in the timing of puberty. Pediatrics 2008; 121(suppl 3):S167-S171.

$\checkmark 8$ Chevalley T, Bonjour JP, Ferrari S, Rizzoli R: The influence of pubertal timing on bone mass acquisition: a predetermined trajectory detectable five years before menarche. J Clin Endocrinol Metab 2009;94:3424-3431.

-9 Hayatbakhsh MR, Najman JM, McGee TR, Bor W, O'Callaghan MJ: Early pubertal maturation in the prediction of early adult substance use: a prospective study. Addiction 2009;104:59-66.

10 Golub MS, Collman GW, Foster PM, Kimmel CA, Rajpert-De Meyts E, Reiter EO, Sharpe RM, Skakkebaek NE, Toppari J: Public health implications of altered puberty timing. Pediatrics 2008;121(suppl 3):S218 S230.

-11 Opdahl S, Alsaker MD, Janszky I, Romundstad PR, Vatten LJ: Joint effects of nulliparity and other breast cancer risk factors. Br J Cancer 2011;105:731-736.

12 Maisonet M, Christensen KY, Rubin C, Holmes A, Flanders WD, Heron J, Ong KK, Golding J, McGeehin MA, Marcus M: Role of prenatal characteristics and early growth on pubertal attainment of British girls. Pediatrics 2010;126:e591-e600.

13 Morris DH, Jones ME, Schoemaker MJ, Ashworth A, Swerdlow AJ: Determinants of age at menarche in the UK: analyses from the Breakthrough Generations Study. Br J Cancer 2010;103:1760-1764.

14 Rubin C, Maisonet M, Kieszak S, Monteilh C, Holmes A, Flanders D, Heron J, Golding J, McGeehin M, Marcus M: Timing of maturation and predictors of menarche in girls enrolled in a contemporary British cohort. Paediatr Perinat Epidemiol 2009;23:492504.

15 Shrestha A, Nohr EA, Bech BH, RamlauHansen CH, Olsen J: Smoking and alcohol use during pregnancy and age of menarche in daughters. Hum Reprod 2011;26:259265.

16 Windham GC, Bottomley C, Birner C, Fenster L: Age at menarche in relation to maternal use of tobacco, alcohol, coffee, and tea during pregnancy. Am J Epidemiol 2004;159: 862-871.

17 Windham GC, Zhang L, Longnecker MP, Klebanoff M: Maternal smoking, demographic and lifestyle factors in relation to daughter's age at menarche. Paediatr Perinat Epidemiol 2008;22:551-561.

18 Ferris JS, Flom JD, Tehranifar P, Mayne ST, Terry MB: Prenatal and childhood environmental tobacco smoke exposure and age at menarche. Paediatr Perinat Epidemiol 2010; 24:515-523.

19 Terry MB, Ferris JS, Tehranifar P, Wei Y, Flom JD: Birth weight, postnatal growth, and age at menarche. Am J Epidemiol 2009;170: 72-79.

20 Sims EK, Addo OY, Gollenberg AL, Himes $\mathrm{JH}$, Hediger ML, Lee PA: Inhibin B and luteinizing hormone levels in girls aged 6-11 years from NHANES III, 1988-1994. Clin Endocrinol (Oxf) 2012;77:555-563.

21 Crofton PM, Evans AE, Groome NP, Taylor MR, Holland CV, Kelnar CJ: Dimeric inhibins in girls from birth to adulthood: relationship with age, pubertal stage, FSH and oestradiol. Clin Endocrinol (Oxf) 2002;56:223230.
22 Chada M, Prusa R, Bronsky J, Pechova M, Kotaska K, Lisa L: Inhibin B, follicle stimulating hormone, luteinizing hormone, and estradiol and their relationship to the regulation of follicle development in girls during childhood and puberty. Physiol Res 2003;52:341-346.

23 Teilmann G, Boas M, Petersen JH, Main KM Gormsen M, Damgaard K, Brocks V, Skakkebaek NE, Jensen TK: Early pituitary-gonadal activation before clinical signs of puberty in 5- to 8-year-old adopted girls: a study of 99 foreign adopted girls and 93 controls. J Clin Endocrinol Metab 2007;92:2538-2544.

24 National Center for Health Statistics: Plan and Operation of the Third National Health and Nutrition Examination Survey, 19881994. Vol Vital Health Stat 1. No 32. Hyattsville, US Department of Health and Human Services, PHS 94-1308, 1994.

25 Gollenberg AL, Hediger ML, Lee PA, Himes $\mathrm{JH}$, Louis GM: Association between lead and cadmium and reproductive hormones in peripubertal U.S. girls. Environ Health Perspect 2010;118:1782-1787.

26 Ludlow H, Muttukrishna S, Hyvonen M, Groome NP: Development of a new antibody to the human inhibin/activin betaB subunit and its application to improved inhibin $B$ ELISAs. J Immunol Methods 2008;329:102111.

27 Robins J, Rotnitzky A, Zhao L: Estimation of regression coefficients when some regressors are not always observed. J Am Stat Assoc 1994;89:846-866.

28 Budtz-Jorgensen E, Grandjean P, Weihe P, White R: Statistical methods for the evaluation of health effects of prenatal mercury exposure. Environmetrics 2003;14:105-120.

29 Greenland S, Brumback B: An overview of relations among causal modelling methods. Int J Epidemiol 2002;31:1030-1037.

30 Davis ME: Structural equation models in occupational health: an application to exposure modelling. Occup Environ Med 2012;69:184190.

$31 \mathrm{Hu}$ L, Bentler P: Cutoff criteria for fit indices in covariance structure analysis: Conventional criteria versus new alternatives structural equation modelling. Struct Equ Modeling 1999;6:1-55.

-32 Nikolov MC, Coull BA, Catalano PJ, Godleski JJ: An informative Bayesian structural equation model to assess source-specific health effects of air pollution. Biostatistics 2007;8:609_ 624 
33 Hart R, Sloboda DM, Doherty DA, Norman RJ, Atkinson HC, Newnham JP, Dickinson JE, Hickey M: Prenatal determinants of uterine volume and ovarian reserve in adolescence. J Clin Endocrinol Metab 2009;94:4931-4937.

34 Baron JA, La Vecchia C, Levi F: The antiestrogenic effect of cigarette smoking in women. Am J Obstet Gynecol 1990;162:502-514.

-35 Windham GC, Mitchell P, Anderson M, Lasley BL: Cigarette smoking and effects on hormone function in premenopausal women. Environ Health Perspect 2005;113:1285-1290.

36 Ibanez L, Potau N, de Zegher F: Ovarian hyporesponsiveness to follicle stimulating hormone in adolescent girls born small for gestational age. J Clin Endocrinol Metab 2000;85: 2624-2626.
37 Ibanez L, Potau N, Enriquez G, de Zegher F: Reduced uterine and ovarian size in adolescent girls born small for gestational age. Pediatr Res 2000;47:575-577.

38 Ibanez L, Potau N, Enriquez G, Marcos MV, de Zegher F: Hypergonadotrophinaemia with reduced uterine and ovarian size in women born small-for-gestational-age. Hum Reprod 2003; 18:1565-1569.

39 Beyerlein A, Ruckinger S, Toschke AM, Schaffrath Rosario A, von Kries R: Is low birth weight in the causal pathway of the association between maternal smoking in pregnancy and higher BMI in the offspring? Eur J Epidemiol 2011;26:413-420.

40 Sorensen K, Mouritsen A, Aksglaede L, Hagen CP, Mogensen SS, Juul A: Recent secular trends in pubertal timing: implications for evaluation and diagnosis of precocious puberty. Horm Res Paediatr 2012;77:137-145.
41 Cortet-Rudelli C, Pigny P, Decanter C, Leroy M, Maunoury-Lefebvre C, Thomas-Desrousseaux P, Dewailly D: Obesity and serum luteinizing hormone level have an independent and opposite effect on the serum inhibin $B$ level in patients with polycystic ovary syndrome. Fertil Steril 2002;77:281-287.

42 De Pergola G, Maldera S, Tartagni M, Pannacciulli N, Loverro G, Giorgino R: Inhibitory effect of obesity on gonadotropin, estradiol, and inhibin B levels in fertile women. Obesity (Silver Spring) 2006;14:1954-1960.

43 Wilkinson JD, Arheart KL, Lee DJ: Accuracy of parental reporting of secondhand smoke exposure: The National Health and Nutrition Examination Survey III. Nicotine Tob Res 2006;8:591-597. 